\title{
Intake of selected foods and beverages and serum uric acid levels in adults: ELSA-Brasil (2008-2010)
}

\author{
Michelle Timóteo da Silva ${ }^{1}$, Maria de Fátima Haueisen Sander Diniz², Carolina \\ Gomes Coelho ${ }^{3}$, Pedro Guatimosim Vidigal ${ }^{4}$, Rosa Weiss Telles ${ }^{2}$ and \\ Sandhi Maria Barreto ${ }^{1,3, *}$ \\ 'Postgraduate Program in Public Health, School of Medicine, Universidade Federal de Minas Gerais, \\ Brazil: ${ }^{2}$ Department of Internal Medicine, School of Medicine, Universidade Federal de Minas Gerais, Brazil: \\ ${ }^{3}$ Department of Preventive Medicine, School of Medicine \& Clinical Hospital, Universidade Federal de Minas Gerais, \\ Brazil: ${ }^{4}$ Post-Graduate Program in Pathology, School of Medicine, Universidade Federal de Minas Gerais, Brazil
}

Submitted 17 September 2018: Final revision received 20 May 2019: Accepted 3 June 2019: First published online 7 October 2019

\begin{abstract}
Objective: To investigate the association between the intake of selected food groups and beverages and serum uric acid (UA).

Design: Cross-sectional study using the baseline data (2008-2010) from the Brazilian Longitudinal Study of Adult Health (ELSA-Brasil). Food intake was assessed by food frequency questionnaire with 114 items. Linear and logistic regressions investigated the associations between the daily intake of each food group (servings/d) and UA (mg/dl) and hyperuricemia (UA $\geq 6.8 \mathrm{mg} / \mathrm{dl}$ ), respectively. All the analyses were adjusted for potential confounders, energy intake and all food groups.

Setting: Teaching and research institutions from six Brazilians states.

Subjects: The participants were 14320 active and retired civil servants, aged 35-74 years.

Results: Higher intake of dairy products was associated with lower serum UA levels in both sexes, with a statistical dose-response gradient. High meat intake was associated with high UA only in women, and high intake of organ meats, in men. Intake of fish and fruits, vegetables and legumes were not associated with serum UA. In men, moderate and high intake of alcoholic beverages, specifically beer and spirits, but not wine, increased UA. In women, only high intake of alcoholic beverages, specifically beer, was associated with increased serum UA. Similar associations were seen for hyperuricemia.

Conclusions: Results suggest a potential beneficial role of dairy products consumption on UA levels. The association between alcohol intake and UA differed according to type of beverage and between sexes. Results reinforce the need to consider the whole diet in the analysis and to conduct sex stratified analysis.
\end{abstract}

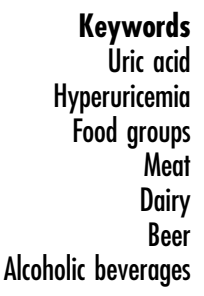

Hyperuricemia, usually defined as serum uric acid (UA) levels over or equal to $6.8 \mathrm{mg} / \mathrm{dl}$, can cause deposition of urate crystals in the joints and periarticular tissues, inducing acute and chronic inflammatory responses and leading to joint damage, a condition known as gout ${ }^{(1,2)}$. Hyperuricemia is a causal component known to be necessary, but not sufficient, for the development of gout ${ }^{(3)}$. Population-based studies have shown a direct, linear or exponential relation between serum UA levels and the incidence of gout ${ }^{(4)}$. Besides gout, hyperuricemia has also been associated with metabolic syndrome, cardiovascular disease, hypertension, obesity, diabetes mellitus and chronic kidney disease (CKD) $)^{(5-10)}$.

Although the relation between diet and hyperuricemia has not yet been elucidated ${ }^{(6)}$, recent studies have shown that diet may have a major influence on serum UA levels ${ }^{(11)}$ both in men and women ${ }^{(12)}$. A study of 4769 elderly patients with high cardiovascular risk found an association between adherence to the Mediterranean diet and lower risk of developing hyperuricemia in 5 years ${ }^{(13)}$. A crosssectional association between high intake of meats and seafood, but not proteins, and higher serum UA levels was 
reported in adults aged 20 years and over in the USA ${ }^{(11)}$. The intake of beer and spirits, but not wine, has been consistently associated with serum UA levels ${ }^{(12,14-16)}$.

Despite the absence of conclusive evidence ${ }^{(17)}$, recommendations to decrease the intake of purine-rich foods, to reduce hyperuricemia are still widely disseminated ${ }^{(12,18,19)}$. It is worth noting, however, that most studies on food intake and serum UA did not perform gender-stratified analysis, or were adjusted for the simultaneous intake of other food groups ${ }^{(12,13,16,20,21)}$. The adjustment for other food groups seems crucial, since we eat a combination of food groups simultaneously and, while these combinations are not at random, they also reflect individual patterns and food choices, and may have different effects on UA production and clearance ${ }^{12}$. The use of medications that may impact serum UA is also a potential confounder in the association between foods and serum UA, and adjustments for these medications are also infrequent in studies on food intake and serum UA levels ${ }^{(12,16,20,21)}$.

This study investigated the association between dietary intake of selected food groups and alcoholic beverages and serum UA levels and the presence of hyperuricemia in subjects of the Brazilian Longitudinal Study of Adult Health (ELSA-Brasil), after adjusting for potential confounders, including the intake of other food groups and alcoholic beverages and the use of medications that can knowingly affect serum UA.

\section{Methods}

\section{Study design}

A cross-sectional study was carried out using baseline data from ELSA-Brasil, which was conducted in 2008-2010. ELSA-Brasil is a multicentre cohort of 15105 active and retired civil servants, aged between 35 and 74 years, from teaching and research institutions located in six states of the country: Minas Gerais, São Paulo, Rio de Janeiro, Espírito Santo, Bahia and Rio Grande do Sul. The main objective of ELSA-Brasil was to investigate the incidence and progression of cardiovascular diseases and diabetes and their social, behavioural, biological and environmental determinants. Data were collected in face-to-face interviews, examinations and standardized measurements conducted by trained and certified examiners ${ }^{(22)}$. ELSA-Brasil was approved by the ethics committee of the six participating institutions and by the National Research Ethics Committee, and all subjects signed an informed consent form. Details on the study design and cohort characteristics have been described in previous publications ${ }^{(22,23)}$.

\section{Study subjects}

This study excluded subjects who: (i) reported using medications to manage hyperuricemia: allopurinol ( $n$ 155) and benzbromarone ( $n 10)$; (ii) with daily energy intake $<2092 \mathrm{~kJ} / \mathrm{d}(500 \mathrm{kcal} / \mathrm{d})$ or $>12104 \mathrm{~kJ} / \mathrm{d}$ $(6000 \mathrm{kcal} / \mathrm{d})(n 208)$; (iii) with a history of bariatric surgery ( $n$ 107); (iv) who missed laboratory UA testing ( $n 5$ ); (v) without information on food intake ( $n$ 395); and (vi) missing on total energy intake ( $n 61)$. Some subjects met more than one exclusion criterion, resulting in 724 subjects excluded. None reported the use of lesinurad, febuxostato, pegloticase or rasburicase and, therefore, these medications were not included in our exclusion criteria.

\section{Data collection and measurements}

\section{Laboratory tests}

UA was measured after a 12-h fasting using a colorimetric enzyme (uricase) method (Siemens ADVIA 1200 system) ${ }^{(24)}$. The glomerular filtration rate (GFR) was estimated based on serum creatinine, sex and age, using the abbreviated Chronic Kidney Disease Epidemiology Collaboration (CKD-EPI) formula, without correction for race ${ }^{(25)}$.

\section{Food intake}

Collected using the semi-quantitative Food Frequency Questionnaire (FFQ), composed of 114 food items, which estimates food intake in the last 12 months. The FFQ provides eight frequency options, that range from 'more than 3 times/d' to 'never/almost never'. The FFQ was shown to be adequate in terms of validity and reproducibility ${ }^{(26)}$.

Three types of alcoholic beverages (beer, wine, and spirits) and five food groups (dairy products; fruits, vegetables and legumes; organ meats; meats; and fish) were analysed in this study. The food items from FFQ and respective serving sizes are described in Supplementary Table S1.

Each food was categorized by the serving size according to the FFQ answers, and servings were multiplied by the frequency, with the following weighting, as previously used in ELSA-Brasil ${ }^{(27)}$ : 3 for $>3$ times/d, 2.5 for 2-3 times/d, 1 for once a day, $0 \cdot 8$ for 5-6 times/week, $0 \cdot 4$ for 2-4 times/week, 0.1 for once a week, 0.07 for 1-3 times/ month and 0 for never/almost never. Then, after multiplying each food by the respective frequency, items in the same group were summed in such a way that the final intake of each group was calculated.

For analysis, the intake categories in servings/d of each food group were defined as 'no/low intake', 'moderate intake' and 'high intake'. For the groups dairy; fruits, vegetables and legumes; meats; and fish, the categories were defined using the intake distribution tertile for men and women, separately. Owing to the high percentage of subjects who did not eat organ meats or drink alcoholic beverages, the category 'no/low intake' corresponded to the absence of intake for these two groups. The moderate and high intake categories were defined by the sex-specific median intake of these foods or beverages among participants who reported consuming these items.

Total energy intake was estimated through the analysis of dietary data of the FFQ, by using the Nutrition Data 
System for Research (NDSR) table and the Brazilian Table of Food Composition (TACO) ${ }^{(26)}$.

\section{Anthropometric measures}

Weight and height were measured in a fasting state. The body mass index (BMI) was calculated dividing the weight (kg) by the squared height $\left(\mathrm{m}^{2}\right)$.

\section{Demographic and behavioural covariates}

Covariates included age (in years), use of medications in the past 2 weeks (losartan, diuretics, acetylsalicylic acid, beta-blockers, and oestrogen for women). All pieces of information were obtained through face-to-face structured interviews carried out by trained, certified professionals under rigorous quality control.

\section{Statistical analysis}

Multiple linear regression was performed to investigate the association between the intake of each food group and serum UA as a continuous variable. Three models were built with successive inclusions: Model 0, unadjusted coefficient; Model 1, which is Model 0 plus age, BMI, energy intake (continuous variable), GFR and use of medications; Model 2 included all previous covariates plus other food groups and alcoholic beverages. We used the log likelihood ratio to test for a linear trend $\left(P_{\text {trend }}\right)$ of categorical variables, by comparing the fitting of two models, the first using the explanatory variable (food group) with its original categories and the second including the same explanatory variable as a continuous one. Values of $P_{\text {trend }}>0.05$ indicate no difference between the two models and support the linear trend hypothesis. The assumptions for performing multiple linear regression were graphically verified.

Finally, we used multiple logistic regression analysis to investigate the association between the intake of each food group and beverage and the presence of hyperuricemia, defined as $\mathrm{UA} \geq 6.8 \mathrm{mg} / \mathrm{dl}$ (no/yes), due to the clinical importance associated with detecting this abnormality. The modelling strategy used in the multiple linear regression was adopted in the logistic analysis.

All analyses were performed using the statistical software Stata 14 with a significance level of $5 \%$ and the $P$-values were two sided.

\section{Results}

A total of 14320 subjects were enrolled in this study, the mean age was $52(\mathrm{SD}=9)$ years, and $55 \%$ were women. The mean serum UA level was $6.4(\mathrm{sD}=1.4) \mathrm{mg} / \mathrm{dl}$ in men and $4.8(\mathrm{SD}=1.2) \mathrm{mg} / \mathrm{dl}$ in women. The prevalence of hyperuricemia was $37 \%$ in men and $6 \%$ in women.

Table 1 presents the study population characteristics according to the serum UA distribution quintile. There was a predominance of men $(82.7 \%)$ in the highest serum UA quintile, and women (91.4\%) in the lowest quintile. The mean age did not seem to be different across serum UA quintiles. The mean BMI was higher in subjects located in the highest serum UA quintile, and the prevalence of hypertension and diabetes progressively increased with increasing serum UA quintile. On the other hand, the mean GFR decreased as serum UA quintile increased.

Table 1 Socio-demographic and health characteristics of ELSA-Brasil baseline participants (2008-2010), according to the serum uric acid distribution quintile

\begin{tabular}{|c|c|c|c|c|c|}
\hline \multirow[b]{2}{*}{ Characteristics } & \multicolumn{5}{|c|}{ Quintile of uric acid (mg/dl) } \\
\hline & $\begin{array}{c}\text { Q1 } \\
(1 \cdot 0-4 \cdot 2)\end{array}$ & $\begin{array}{c}\text { Q2 } \\
(4 \cdot 3-5 \cdot 0)\end{array}$ & $\begin{array}{c}\text { Q3 } \\
(5 \cdot 1-5 \cdot 8)\end{array}$ & $\begin{array}{c}\text { Q4 } \\
(5 \cdot 9-6 \cdot 7)\end{array}$ & $\begin{array}{c}\text { Q5 } \\
(6 \cdot 8-13 \cdot 4)\end{array}$ \\
\hline \multicolumn{6}{|l|}{ Age (years) } \\
\hline Mean & 50 & 51 & 53 & 53 & 53 \\
\hline SD & $8 \cdot 7$ & 8.9 & $9 \cdot 2$ & $9 \cdot 0$ & $9 \cdot 2$ \\
\hline \multicolumn{6}{|l|}{ Sex $(\%)$} \\
\hline Men & 8.6 & $25 \cdot 7$ & $45 \cdot 8$ & 65.4 & $82 \cdot 7$ \\
\hline Women & 91.4 & $74 \cdot 3$ & $54 \cdot 2$ & 34.6 & $17 \cdot 3$ \\
\hline \multicolumn{6}{|l|}{ Educational level (\%) } \\
\hline Incomplete elementary school & 3.9 & 4.4 & 5.8 & $6 \cdot 8$ & 8.5 \\
\hline Complete elementary school & 4.8 & $6 \cdot 2$ & 6.4 & 7.4 & $9 \cdot 2$ \\
\hline High school & 34.6 & 32.6 & 35.5 & 33.9 & 34.7 \\
\hline University degree & $56 \cdot 6$ & $56 \cdot 9$ & $52 \cdot 2$ & 51.9 & 47.5 \\
\hline \multicolumn{6}{|l|}{ BMl $\left(\mathrm{kg} / \mathrm{m}^{2}\right)$} \\
\hline Mean & 24.9 & $26 \cdot 2$ & $27 \cdot 1$ & 27.9 & 28.9 \\
\hline SD & $4 \cdot 1$ & 4.5 & 4.6 & 4.6 & 4.8 \\
\hline Hypertension (\%) & 21.9 & $28 \cdot 2$ & 34.7 & $40 \cdot 0$ & 53.5 \\
\hline Diabetes (\%) & 10.5 & $15 \cdot 9$ & 18.9 & 23.0 & 29.5 \\
\hline \multicolumn{6}{|l|}{ GFR $\left(\mathrm{ml} / \mathrm{min} / 1.73 \mathrm{~m}^{2}\right)$} \\
\hline Mean & 91.6 & 87.6 & 85.2 & 84.0 & 78.9 \\
\hline SD & 13.9 & $14 \cdot 8$ & $14 \cdot 4$ & 14.4 & $15 \cdot 8$ \\
\hline
\end{tabular}

Q, quintile. GFR, glomerular filtration rate. 
Food, beverages and uric acid in ELSA-Brasil

Table 2 Intake categories in servings/d of the food groups used in the present study in ELSA-Brasil baseline participants (2008-2010)

\begin{tabular}{|c|c|c|c|c|}
\hline & $\begin{array}{l}\text { Frequency of } \\
\text { non-intake }(n)\end{array}$ & $\begin{array}{l}\text { No/low intake } \\
\text { (servings/d) }\end{array}$ & $\begin{array}{l}\text { Moderate intake } \\
\text { (servings/d) }\end{array}$ & $\begin{array}{l}\text { High intake } \\
\text { (servings/d) }\end{array}$ \\
\hline \multicolumn{5}{|l|}{ Dairy } \\
\hline$M$ & 61 & $0 a \leq 2.18$ & $>2.18 a \leq 4.30$ & $>4.30$ \\
\hline $\mathrm{F}$ & 41 & $0 a \leq 2.53$ & $>2.53 a \leq 4.57$ & $>4.57$ \\
\hline \multicolumn{5}{|c|}{ Fruits, vegetables and legumes } \\
\hline M & 1 & $0 \mathrm{a} \leq 10.04$ & $>10.04 a \leq 17.15$ & $>17 \cdot 15$ \\
\hline $\mathrm{F}$ & 0 & $0 \mathrm{a} \leq 10.76$ & $>10.76 a \leq 17.84$ & $>17.84$ \\
\hline \multicolumn{5}{|c|}{ Meats } \\
\hline M & 65 & $0 a \leq 1.34$ & $>1.34 \mathrm{a} \leq 2.40$ & $>2.40$ \\
\hline $\mathrm{F}$ & 130 & $0 a \leq 1.05$ & $>1.05 a \leq 1.80$ & $>1.80$ \\
\hline \multicolumn{5}{|c|}{ Organ meats } \\
\hline$M$ & 3496 & 0 & $>0 a \leq 0.14$ & $>0.14$ \\
\hline $\mathrm{F}$ & 5097 & 0 & $>0 \mathrm{a} \leq 0.10$ & $>0.10$ \\
\hline \multicolumn{5}{|l|}{ Fish } \\
\hline M & 785 & $0 a \leq 0.20$ & $>0.20 a \leq 0.45$ & $>0.45$ \\
\hline $\mathrm{F}$ & 906 & $0 \mathrm{a} \leq 0.14$ & $>0.14 \mathrm{a} \leq 0.40$ & $>0.40$ \\
\hline \multicolumn{5}{|c|}{ Alcoholic beverages } \\
\hline M & 1723 & 0 & $>0 a \leq 0.98$ & $>0.98$ \\
\hline $\mathrm{F}$ & 3618 & 0 & $>0 \mathrm{a} \leq 0.34$ & $>0.34$ \\
\hline \multicolumn{5}{|l|}{ Beer } \\
\hline M & 2398 & 0 & $>0 a \leq 0.80$ & $>0.80$ \\
\hline $\mathrm{F}$ & 4933 & 0 & $>0 \mathrm{a} \leq 0.29$ & $>0.29$ \\
\hline \multicolumn{5}{|l|}{ Wine } \\
\hline M & 3398 & 0 & $>0 \mathrm{a} \leq 0.20$ & $>0.20$ \\
\hline $\mathrm{F}$ & 4778 & 0 & $>0 \mathrm{a} \leq 0.14$ & $>0.14$ \\
\hline \multicolumn{5}{|c|}{ Spirits } \\
\hline$M$ & 4813 & 0 & $>0 a \leq 0.14$ & $>0.14$ \\
\hline $\mathrm{F}$ & 7273 & 0 & $>0 a \leq 0.10$ & $>0.10$ \\
\hline
\end{tabular}

M, males. F, females.

Table 2 shows the intake categories in servings/d for each food group and alcoholic beverages, according to the intake categories defined previously. The results of the multiple linear regression show statistically significant associations with a dose-response gradient between higher intake of dairy products and lower mean serum UA levels for men $\left(P_{\text {trend }}=0.131\right)$ and women $\left(P_{\text {trend }}=0.349\right)($ Table 3$)$. The mean serum UA was $0.22 \mathrm{mg} / \mathrm{dl}(\mathrm{CI} 95 \%-0 \cdot 30,-0 \cdot 14)$ lower for men and $0.17 \mathrm{mg} / \mathrm{dl}(\mathrm{CI} 95 \%-0 \cdot 23,-0 \cdot 11)$ lower for women in the high dairy intake groups compared with the no/low intake groups.

We did not find any significant association between the intake of fruits, vegetables and legumes, and serum UA after adjustments (Table 3).

Only high consumption of meats was statistically associated with higher mean serum UA levels in both sexes, but this association remained statistically significant only among women in Model 2. After all adjustments, men with high intake of organ meats presented significantly higher mean serum UA than men with no/low intake. No significant association was found between the intake of fish and serum UA levels in women. In men, high fish intake was associated with higher mean serum UA levels, but only in models 0 and 1 (Table 3).

Higher intake of alcoholic beverages showed strong association with elevated serum UA levels in all three models for men, with no evidence of dose response gradient $\left(P_{\text {trend }}=0.008\right)$. In the fully adjusted analysis, the mean serum UA was $0.47 \mathrm{mg} / \mathrm{dl}$ (CI $95 \% 0.39,0.55)$ greater in the high intake group of alcoholic beverages compared with the no/low intake group. Among women, only the high intake group of alcoholic beverages was associated with an increase in mean serum UA in the final analysis (Table 3). When assessed by type of beverage, significant associations were observed between increasing intake of beer and higher mean serum UA levels in men and women, after adjusting for all potential confounders, with the support of a linear trend in the association among women $\left(P_{\text {trend }}=0 \cdot 171\right)$, but not among men $\left(P_{\text {trend }}=0.05\right)$. Moderate and high intakes of wine were associated with lower mean serum UA in women in Model 0, but this association lost significance after the adjustments. In men, high intake of wine remained associated with higher mean serum UA after considering age, BMI, GFR, and use of medications, but this association lost statistical significance in Model 2. In men, only the high intake of spirits remained statistically associated with higher mean serum UA levels in Model 2 $(\beta=0.20$; CI $95 \% 0.11,0.29)$. In women, the high intake of spirits increased the mean serum UA levels in Model 1, but lost statistical significance in Model 2 (Table 3).

In the logistic regression analysis considering the presence of hyperuricemia (Table 4), greater intake of dairy products persisted associated with lower chances of hyperuricemia in men, with a downward linear trend $\left(P_{\text {trend }}=0.703\right)$. In women, only the high intake of dairy remained associated with a lower chance of hyperuricemia $(\mathrm{OR}=0.71$; CI $95 \% 0.54,0.93)$. No association was found between the intake of fruits, vegetables and legumes and 
Table 3 Multiple linear regression analysis between daily consumption of food and alcoholic beverages in intake categories $\dagger$ and serum uric acid levels $(\mathrm{mg} / \mathrm{dl})(\mathrm{N}=14,320)$

\begin{tabular}{|c|c|c|c|c|c|c|c|c|c|c|c|c|}
\hline & \multicolumn{6}{|c|}{$\operatorname{Men}(N=6433)$} & \multicolumn{6}{|c|}{ Women $(N=7887)$} \\
\hline & \multicolumn{2}{|c|}{ Model 0} & \multicolumn{2}{|c|}{ Model 1} & \multicolumn{2}{|c|}{ Model 2} & \multicolumn{2}{|c|}{ Model 0} & \multicolumn{2}{|c|}{ Model 1} & \multicolumn{2}{|c|}{ Model 2} \\
\hline & $\beta$ & Cl $95 \%$ & $\beta$ & Cl $95 \%$ & $\beta$ & Cl $95 \%$ & $\beta$ & Cl $95 \%$ & $\beta$ & Cl $95 \%$ & $\beta$ & Cl $95 \%$ \\
\hline \multicolumn{13}{|l|}{ Dairy $\ddagger \S$} \\
\hline No/low intake & 0 & & 0 & & 0 & & 0 & & 0 & & 0 & \\
\hline Moderate intake & $-0 \cdot 19^{\star \star}$ & $-0.28,-0.11$ & $-0 \cdot 19^{\star \star}$ & $-0.26,-0.11$ & $-0 \cdot 16^{\star \star}$ & $-0.24,-0.08$ & $-0 \cdot 14^{\star \star}$ & $-0.21,-0.08$ & $-0 \cdot 11^{\star *}$ & $-0.17,-0.06$ & $-0.11^{\star \star}$ & $-0.16,-0.05$ \\
\hline High intake & $-0 \cdot 27^{\star *}$ & $-0.35,-0 \cdot 18$ & $-0 \cdot 27^{\star \star}$ & $-0.36,-0.19$ & $-0 \cdot 22^{\star *}$ & $-0.30,-0.14$ & $-0 \cdot 20^{* *}$ & $-0.27,-0.14$ & $-0 \cdot 18^{* *}$ & $-0.24,-0.12$ & $-0 \cdot 17^{\star *}$ & $-0.23,-0.11$ \\
\hline \multicolumn{13}{|c|}{ Fruits, vegetables and legumes } \\
\hline No/low intake & 0 & & 0 & & 0 & & 0 & & 0 & & 0 & \\
\hline Moderate intake & -0.01 & $-0.08,0.08$ & -0.02 & $-0.10,0.06$ & -0.01 & $-0.08,0.07$ & $0.09^{*}$ & $0.03,0.16$ & 0.01 & $-0.05,0.06$ & 0.01 & $-0.04,0.07$ \\
\hline High intake & 0.03 & $-0.05,0.12$ & -0.05 & $-0.13,0.04$ & -0.01 & $-0.09,0.07$ & $0 \cdot 16^{\star \star}$ & $0.10,0.23$ & 0.01 & $-0.05,0.07$ & 0.01 & $-0.05,0.07$ \\
\hline \multicolumn{13}{|l|}{ Meats } \\
\hline No/low intake & 0 & & 0 & & 0 & & 0 & & 0 & & 0 & \\
\hline Moderate intake & $0 \cdot 16^{\star *}$ & $0.08,0.25$ & $0.09^{*}$ & $0.01,0.16$ & 0.04 & $-0.04,0.12$ & 0.03 & $-0.04,0.09$ & 0.05 & $-0.01,0.10$ & 0.04 & $-0.02,0.09$ \\
\hline High intake & $0.24^{\star \star}$ & $0.16,0.33$ & $0.14^{\star}$ & $0.05,0.23$ & 0.08 & $-0.01,0.17$ & $0 \cdot 20^{\star \star}$ & $0.14,0.26$ & $0.11^{\star \star}$ & $0.05,0.17$ & $0.08^{\star}$ & $0.02,0.14$ \\
\hline \multicolumn{13}{|l|}{ Organ meats } \\
\hline No/low intake & 0 & & 0 & & 0 & & 0 & & 0 & & 0 & \\
\hline Moderate intake & 0.01 & $-0.09,0.10$ & 0.07 & $-0.02,0.16$ & 0.02 & $-0.06,0.11$ & 0.05 & $-0.03,0.13$ & 0.04 & $-0.03,0.10$ & 0.02 & $-0.04,0.09$ \\
\hline High intake & $0 \cdot 21^{\star *}$ & $0.13,0.29$ & $0.20^{\star *}$ & $0.12,0.27$ & $0.09^{*}$ & $0.02,0.17$ & $0.09^{*}$ & $0.02,0.16$ & 0.01 & $-0.05,0.06$ & -0.04 & $-0.10,0.02$ \\
\hline \multicolumn{13}{|l|}{ Fish } \\
\hline No/low intake & 0 & & 0 & & 0 & & 0 & & 0 & & 0 & \\
\hline Moderate intake & 0.05 & $-0.03,0.13$ & $0.08^{\star}$ & $0.01,0.16$ & 0.05 & $-0.03,0.12$ & -0.03 & $-0.10,0.03$ & -0.01 & $-0.06,0.05$ & -0.01 & $-0.07,0.04$ \\
\hline High intake & $0.13^{*}$ & $0.04,0.21$ & $0.12^{*}$ & $0.04,0.20$ & 0.05 & $-0.03,0.13$ & -0.01 & $-0.07,0.07$ & 0.04 & $-0.02,0.10$ & 0.02 & $-0.04,0.08$ \\
\hline \multicolumn{13}{|l|}{ Alcoholic beverages } \\
\hline No/low intake & 0 & & 0 & & 0 & & 0 & & 0 & & 0 & \\
\hline Moderate intake & 0.12 & $0.03,0.21$ & $0.14^{* *}$ & $0.06,0.22$ & $0.15^{\star *}$ & $0.07,0.23$ & $-0 \cdot 13^{\star *}$ & $-0.20,-0.07$ & -0.02 & $-0.07,0.04$ & -0.01 & $-0.07,0.04$ \\
\hline High intake & $0.48^{\star \star}$ & $0.39,0.56$ & $0.49^{\star \star}$ & $0.41,0.57$ & $0.47^{\star \star}$ & $0.39,0.55$ & 0.02 & $-0.04,0.09$ & $0 \cdot 16^{\star \star}$ & $0.10,0.21$ & $0 \cdot 16^{\star \star}$ & $0.10,0.21$ \\
\hline \multicolumn{13}{|l|}{ Beer $§$} \\
\hline No/low intake & 0 & & 0 & & 0 & & 0 & & 0 & & 0 & \\
\hline Moderate intake & $0 \cdot 16^{\star \star}$ & $0.07,0.24$ & $0.15^{\star \star}$ & $0.08,0.23$ & $0.12^{\star}$ & $0.04,0.20$ & -0.03 & $-0.10,0.04$ & 0.05 & $-0.01,0.11$ & $0.07^{\star}$ & $0.01,0.13$ \\
\hline High intake & $0.49^{\star *}$ & $0.41,0.57$ & $0.48^{\star *}$ & $0.41,0.56$ & $0.38^{\star *}$ & $0.30,0.47$ & $0.13^{\star \star}$ & $0.06,0.20$ & $0.22^{\star *}$ & $0.16,0.28$ & $0.22^{* *}$ & $0.16,0.28$ \\
\hline \multicolumn{13}{|l|}{ Wine } \\
\hline No/low intake & 0 & & 0 & & 0 & & 0 & & 0 & & 0 & \\
\hline Moderate intake & -0.04 & $-0.13,0.04$ & 0.01 & $-0.08,0.08$ & -0.05 & $-0.13,0.03$ & $-0 \cdot 11^{*}$ & $-0.18,-0.04$ & -0.01 & $-0.07,0.04$ & -0.03 & $-0.09,0.03$ \\
\hline High intake & $0.13^{*}$ & $0.04,0.21$ & $0.13^{\star *}$ & $0.05,0.21$ & 0.02 & $-0.06,0.11$ & $-0 \cdot 14^{\star *}$ & $-0.20,-0.07$ & 0.02 & $-0.04,0.08$ & -0.04 & $-0.11,0.02$ \\
\hline \multicolumn{13}{|l|}{ Spirit } \\
\hline No/low intake & 0 & & 0 & & 0 & & 0 & & 0 & & 0 & \\
\hline Moderate intake & $0 \cdot 18^{*}$ & $0.07,0.30$ & $0.19^{\star \star}$ & $0.09,0.29$ & 0.09 & $-0.02,0.20$ & $-0.19^{*}$ & $-0.32,-0.05$ & -0.05 & $-0.17,0.07$ & -0.05 & $-0.17,0.07$ \\
\hline High intake & $0.38^{\star \star}$ & $0.29,0.48$ & $0.37^{\star \star}$ & $0.28,0.46$ & $0.20^{\star *}$ & $0.11,0.29$ & 0.06 & $-0.07,0.19$ & $0.11^{*}$ & $0.01,0.22$ & 0.08 & $-0.04,0.19$ \\
\hline
\end{tabular}

$\beta$, coefficient $\beta$.Model 1: adjusted for age, body mass index, glomerular filtration rate, use of medications (losartan, diuretics, acetylsalicylic acid, beta-blockers, and oestrogen for women).

Model 2: additionally, adjusted by the other groups of foods and alcoholic beverages.

${ }^{*} P$-value $<0.05$.

${ }^{\star *} P$-value $<0.001$.

IIntake categories shown in Table 2 .
¥Model 2 Log likelihood ratio test for men: $P_{\text {trend }}>0.05$

†Model 2 Log likelihood ratio test for men: $P_{\text {trend }}>0.05$.
§Model 2 Log likelihood ratio test for women: $P_{\text {trend }}>0.05$ 


\section{Public Health Nutrition}

Table 4 Association between daily consumption of food and alcoholic beverages in intake categories $†$ and the presence of hyperuricemiał $(N=14,320)$

\begin{tabular}{|c|c|c|c|c|c|c|c|c|c|c|c|c|}
\hline & \multicolumn{6}{|c|}{ Men $(N=6433)$} & \multicolumn{6}{|c|}{ Women $(N=7887)$} \\
\hline & \multicolumn{2}{|c|}{ Model 0} & \multicolumn{2}{|c|}{ Model 1} & \multicolumn{2}{|c|}{ Model 2} & \multicolumn{2}{|c|}{ Model 0} & \multicolumn{2}{|c|}{ Model 1} & \multicolumn{2}{|c|}{ Model 2} \\
\hline & OR & $\mathrm{Cl} 95 \%$ & OR & $\mathrm{Cl} 95 \%$ & OR & $\mathrm{Cl} 95 \%$ & OR & $\mathrm{Cl} 95 \%$ & OR & $\mathrm{Cl} 95 \%$ & OR & $\mathrm{Cl} 95 \%$ \\
\hline \multicolumn{13}{|l|}{ Dairy§ } \\
\hline No/low intake & 1 & & 1 & & 1 & & 1 & & 1 & & 1 & \\
\hline Moderate intake & $0.84^{*}$ & $0.74,0.94$ & $0.81^{*}$ & $0.71,0.93$ & $0.85^{\star}$ & $0.74,0.97$ & $0.77^{*}$ & $0.62,0.95$ & $0.79^{*}$ & $0.62,0.99$ & 0.82 & $0.64,1.05$ \\
\hline High intake & $0 \cdot 70^{\star *}$ & $0.62,0.79$ & $0 \cdot 63^{\star \star}$ & $0.55,0.73$ & $0.68^{\star *}$ & $0.60,0.79$ & $0 \cdot 64^{\star *}$ & $0.51,0.80$ & $0 \cdot 65^{\star \star}$ & $0.54,0.84$ & $0.71^{*}$ & $0.54,0.93$ \\
\hline \multicolumn{13}{|c|}{ Fruits, vegetables and legumes } \\
\hline No/low intake & 1 & & 1 & & 1 & & 1 & & 1 & & 1 & \\
\hline Moderate intake & 0.97 & $0.86,1 \cdot 10$ & 0.93 & $0.82,1.07$ & 0.96 & $0.84,1 \cdot 11$ & 1.24 & $0.99,1.56$ & 1.07 & $0.83,1.38$ & 1.07 & $0.83,1.39$ \\
\hline High intake & 1.05 & $0.93,1.19$ & 0.91 & $0.78,1.05$ & 0.97 & $0.84,1 \cdot 12$ & $1 \cdot 26^{*}$ & $1.01,1.58$ & 0.96 & $0.73,1.25$ & 0.98 & $0.74,1.28$ \\
\hline \multicolumn{13}{|l|}{ Meats } \\
\hline No/low intake & 1 & & 1 & & 1 & & 1 & & 1 & & 1 & \\
\hline Moderate intake & $1 \cdot 27^{\star \star}$ & $1.12,1.45$ & $1 \cdot 19^{\star}$ & $1.04,1.37$ & $1 \cdot 11$ & $0.96,1.28$ & 1.05 & $0.83,1.33$ & $1 \cdot 26$ & $0.97,1.64$ & 1.23 & $0.94,1.61$ \\
\hline High intake & $1.49^{\star \star}$ & $1.32,1.69$ & $1 \cdot 37^{\star \star}$ & $1.17,1.61$ & $1 \cdot 25^{\star}$ & $1.06,1.47$ & $1.49^{\star \star}$ & $1 \cdot 19,1.86$ & $1.58^{\star}$ & $1 \cdot 19,2 \cdot 10$ & $1.47^{\star}$ & $1.10,1.96$ \\
\hline \multicolumn{13}{|l|}{ Organ meats } \\
\hline No/low intake & 1 & & 1 & & 1 & & 1 & & 1 & & 1 & \\
\hline Moderate intake & 1.06 & $0.92,1.22$ & $1 \cdot 16^{*}$ & $0.99,1.36$ & 1.09 & $0.93,1.28$ & 1.05 & $0.81,1.37$ & 1.09 & $0.82,1.46$ & 1.04 & $0.78,1.40$ \\
\hline High intake & $1 \cdot 29^{\star \star}$ & $1 \cdot 15,1 \cdot 45$ & $1 \cdot 30^{\star *}$ & $1 \cdot 14,1.48$ & $1 \cdot 10$ & $0.97,1.26$ & $1 \cdot 25^{\star}$ & $1.00,1.55$ & $1 \cdot 12$ & $0.87,1.43$ & 0.98 & $0.76,1.26$ \\
\hline \multicolumn{13}{|l|}{ Fish } \\
\hline No/low intake & 1 & & 1 & & 1 & & 1 & & 1 & & 1 & \\
\hline Moderate intake & 1.08 & $0.95,1.23$ & $1 \cdot 14^{*}$ & $0.99,1.31$ & 1.08 & $0.94,1.25$ & 0.98 & $0 \cdot 78,1 \cdot 24$ & 1.05 & $0.81,1.35$ & 1.02 & $0.79,1.32$ \\
\hline High intake & $1 \cdot 17^{*}$ & $1.03,1.33$ & $1 \cdot 16^{*}$ & $1.00,1.33$ & 1.05 & $0.90,1.22$ & $1 \cdot 10$ & $0.87,1.40$ & $1 \cdot 21$ & $02 \cdot 92,1.59$ & $1 \cdot 18$ & $0.89,1.56$ \\
\hline \multicolumn{13}{|l|}{ Alcoholic beverages§ } \\
\hline No/low intake & 1 & & 1 & & 1 & & 1 & & 1 & & 1 & \\
\hline Moderate intake & $1 \cdot 20$ & $1.05,1.37$ & $1 \cdot 31^{\star \star}$ & $1 \cdot 13,1.51$ & $1 \cdot 33^{\star *}$ & $1.15,1.54$ & $0 \cdot 66^{\star \star}$ & $0.52,0.84$ & 0.84 & $0.64,1.08$ & 0.84 & $0.65,1 \cdot 10$ \\
\hline High intake & $1.91^{\star \star}$ & $1 \cdot 67,2 \cdot 17$ & $2 \cdot 20^{\star \star}$ & $1.90,2.54$ & $2 \cdot 16^{\star \star}$ & $1.86,2.50$ & 0.94 & $0.76,1.16$ & $1.42^{\star}$ & $1.12,1.80$ & $1.40^{\star}$ & $1.10,1.78$ \\
\hline \multicolumn{13}{|l|}{ Beer§ } \\
\hline No/low intake & 1 & & 1 & & 1 & & 1 & & 1 & & 1 & \\
\hline Moderate intake & $1 \cdot 24^{\star *}$ & $1.09,1.41$ & $1 \cdot 29^{* *}$ & $1.12,1.48$ & $1 \cdot 23^{*}$ & $1.06,1.43$ & 0.86 & $0.67,1.11$ & 1.02 & $0.77,1.35$ & 1.08 & $0.80,1.45$ \\
\hline High intake & $1.98^{\star \star}$ & $1 \cdot 74,2 \cdot 22$ & $2 \cdot 19^{\star \star}$ & $1.92,2.51$ & $1 \cdot 89^{\star *}$ & $1 \cdot 63,2 \cdot 20$ & 1.23 & $0.98,1.54$ & $1 \cdot 80^{\star *}$ & $1 \cdot 40,2 \cdot 31$ & $1 \cdot 82^{\star *}$ & $1 \cdot 39,2 \cdot 38$ \\
\hline \multicolumn{13}{|l|}{ Wine } \\
\hline No/low intake & 1 & & 1 & & 1 & & 1 & & 1 & & 1 & \\
\hline Moderate intake & 0.94 & $0.83,1.07$ & 1.00 & $0.87,1 \cdot 15$ & 0.93 & $0.80,1.08$ & $0 \cdot 81$ & $0.63,1.04$ & 0.99 & $0.76,1.30$ & 0.95 & $0.71,1.26$ \\
\hline High intake & $1 \cdot 20^{*}$ & $1.06,1.35$ & $1 \cdot 24^{*}$ & $1.08,1.41$ & 1.06 & $0.91,1.23$ & $0 \cdot 68^{*}$ & $0.53,0.88$ & 0.96 & $0 \cdot 73,1 \cdot 26$ & $0 \cdot 81$ & $0.60,1.10$ \\
\hline \multicolumn{13}{|l|}{ Spirit } \\
\hline No/low intake & 1 & & 1 & & 1 & & 1 & & 1 & & 1 & \\
\hline Moderate intake & $1 \cdot 27^{\star}$ & $1.08,1.51$ & $1 \cdot 35^{\star \star}$ & $1 \cdot 13,1.62$ & $1 \cdot 14$ & $0.94,1.39$ & 0.71 & $0.41,1.22$ & 0.98 & $0.54,1.76$ & 0.98 & $0.53,1.81$ \\
\hline High intake & $1.69^{\star \star}$ & $1.48,1.94$ & $1.79^{\star \star}$ & $1.54,2.08$ & $1.37^{\star \star}$ & $1.16,1.61$ & 1.02 & $0.66,1.59$ & $1 \cdot 17$ & $0.72,1.90$ & $1 \cdot 11$ & $0.67,1.84$ \\
\hline
\end{tabular}

Model 1: adjusted for age, body mass index, glomerular filtration rate, use of medications (losartan, diuretics, acetylsalicylic acid, beta-blockers, and oestrogen for women).

Model 2: additionally, adjusted by the other groups of foods and alcoholic beverages.

${ }^{*} P$-value $<0.05$.

P-value $<0.001$.

Tawn in Table 2

SModel 2 Log likelihood ratio test for men: $P_{\text {trend }}>0.05$ 
hyperuricemia, after considering potential confounders. Only the high intake of meats was independently associated with increased odds of hyperuricemia in both men and women in the final analysis. Neither organ meats nor fish consumption was associated with hyperuricemia after adjustments.

After considering all confounders, greater intake of alcohol was associated with hyperuricemia in men with a doseresponse gradient $\left(P_{\text {trend }}=0 \cdot 103\right)$, giving the high intake group a twofold increase in the chances of hyperuricemia when compared with no/low intake group. Among women, the association was only significant for the high intake of alcohol, increasing the chances of hyperuricemia by $40 \%$ as compared with the no/low intake (Table 4). In men, beer intake was associated with higher chances of hyperuricemia, showing a dose-response gradient $\left(P_{\text {trend }}=0.086\right)$, after adjusting for potential confounders. Women in the high intake group of beer were nearly two times more likely to have hyperuricemia after taking into account all potential confounders. Only in men was the high intake of wine associated with a higher chance of hyperuricemia, but this association lost statistical significance in Model 2. After all adjustments, men in the high intake category of spirits were $37 \%$ more likely to have hyperuricemia than the no/low intake. For women, no association was found between the intake of spirits and hyperuricemia (Table 4).

\section{Discussion}

This study investigated the association between five food groups and alcoholic beverages and serum UA levels in men and women enrolled at baseline in a large cohort of Brazilian adults. After adjusting for demographic, anthropometric, behavioural, health condition and laboratory variables, as well as for the intake of other food groups, alcoholic beverages and use of medications, serum UA levels showed an inverse association with dairy intake in both sexes and a direct association with intake of beer in men, with indications of a dose-response gradient in these associations. In addition, high consumption of meats and beer in women, and organ meats in men, were also associated with higher serum UA levels. Similar results were obtained in the analysis that considered the presence of hyperuricemia (UA $\geq 6.8 \mathrm{mg} / \mathrm{dl}$ ).

Our results support previous evidence indicating that the intake of dairy products decreases serum $\mathrm{UA}^{(11,12,15,17,28)}$. One of the main explanations for this finding is that the protein fraction of dairy products has antioxidant properties, and UA is one of the oxidants ${ }^{(29)}$. In an experimental study with ten subjects, milk proteins casein and lactoalbumin decreased serum UA levels in healthy subjects $^{(30)}$. However, the effects of orotic acid in milk which promotes renal urate excretion, and the uricosuric effect of milk casein and lactalbumin on serum UA have yet to be confirmed ${ }^{(29)}$.
The intake of fruits, vegetables and legumes was not statistically associated with serum UA, in line with previous studies $^{(15,17,19,21)}$. A higher intake of fruits was associated with lower serum UA levels in the AusDiab Study and Troms $\varnothing$ Study cohorts but the authors did not find any significant relationship between the consumption of legumes and serum UA in any of the two cohorts ${ }^{(12)}$.

Different from some results ${ }^{(11,13)}$, but in line with most previous studies ${ }^{(12,17,31)}$, we did not find any association between the intake of fish and serum UA or hyperuricemia. The population of the ELSA-Brasil study includes subjects from four coastal cities in Brazil, where fish consumption is high. Moreover, neither of the two studies mentioned above, which found an association between fish consumption and serum UA, made any adjustments for the intake of other food groups.

In this study, high intake of meat was statistically associated with higher chances of hyperuricemia in both sexes, but the association with serum UA was statistically significant only in women after adjustments, showing borderline $P$-value in men $(P=0.09)$. No studies conducting a similar sex-stratified analysis were found. Some studies found an association between greater intake of meat and increased serum UA levels ${ }^{(11,12)}$, and others found no association between these variables ${ }^{(21,28)}$. Therefore, this association seems to be controversial in the literature. A previous study $^{(13)}$ found that individuals with low intake of meats (<1 serving/d) had $23 \%$ lower risk of hyperuricemia in a longitudinal analysis when compared with individuals with high intake of this food group ${ }^{(13)}$. While this finding is in the opposite direction of what we investigated here (high consumption as a risk factor), we believe that the above quoted longitudinal finding is in line with our results, indicating that high consumption of meats may increase the chances of hyperuricemia in men and women.

High intake of organ meats did not remain associated with hyperuricemia in either sex, but was associated with serum UA only in men after adjustments. It is important to stress that, on average, women eat considerably less organ meats than men, and this might explain while we found no association among women. Although they are usually included in recommendations for serum UA control $^{(32)}$, this seems to be justified only by the high content of purines present in organ meats ${ }^{(18)}$, with no previous studies supporting the diet recommendation ${ }^{(32)}$. Thus, our results contribute to reversing this lack of evidence in the literature.

Our results support other previous studies that found an association between higher beer intake and higher serum UA levels ${ }^{(12,14-16)}$. However, our findings suggest that the consumption of spirits is associated with serum UA and hyperuricemia only in men. Most studies on alcohol intake and serum UA did not perform any sex-stratified analysis. A study analysed the cross-sectional and longitudinal associations between intake of alcoholic beverages and serum UA and found stronger cross-sectional associations in 
women than in men, but no longitudinal association ${ }^{(28)}$. The explanations for the association with alcohol beverage include the fact that alcohol increases UA production in the liver through degradation of adenosine triphosphate ${ }^{(28)}$ and decreases urate excretion due to dehydration and metabolic acidosis, which may occur when alcohol is consumed in more substantial amounts ${ }^{(33)}$. Furthermore, beer alone has a high content of purines and, depending on the amount drank, it could impact serum UA levels ${ }^{(34)}$. The large amount of beer intake in men in the current study may explain why we have not found a dose-response gradient between AU and beer intake in this subgroup.

In our study, wine intake was not associated with either serum UA or hyperuricemia after adjustments, in line with the results of other studies ${ }^{(12,14,15)}$. However, in a Spanish cohort of 4449 elderly participants at high risk for cardiovascular disease, the intake of seven or more glasses of wine per week was associated with an increased risk of hyperuricemia ${ }^{(13)}$, but this level of intake is very high, and much higher than that observed among ELSA-Brasil participants.

It is important to note that, when assessed as one single group, alcoholic beverages were associated with hyperuricemia, possibly because beer consumption is predominant in the population studied.

The strengths of this study include the sample size, which allowed for separate analyses of men and women, the rigorous data collection, the standardization of how examinations were performed, and adjustments for important confounders, such as GFR, use of medications and intake of other food groups and alcoholic beverages. In addition, the analysis considering hyperuricemia as the response variable is also an additional contribution, as changes in the mean value of serum UA, as observed in the linear regression analysis, may not have clinical significance, which is different from hyperuricemia.

Our results show that adjusting for other food groups and alcoholic beverages had an impact on final results. Such adjustments are infrequent in the literature and some of them attenuated associations between serum AU and food groups, such as for dairy and beer in the linear regression in men for some food groups in our study. The combination of different foods affects the bioavailability of nutrients as well as purine metabolism and may influence UA production and excretion, and thus affect serum UA levels ${ }^{(35)}$. It is already known that food combinations are conditioned to individual patterns and food choices, and there are marked differences between the sexes ${ }^{(36)}$. We believe that the lack of these adjustments can explain some inconsistencies among the associations found in the literature.

The limitations of this study includes the difficulty in accurately estimating food intake from a food frequency questionnaire. Although validated, this method is not as accurate as the daily food record ${ }^{(37)}$. Despite having adjusted for the food groups assessed, we know that dietary patterns are involved, and these adjustments might be insufficient to deal with the complexity of dietary combinations. Finally, this is a cross-sectional study, and it is not possible to show a causal relation between explanatory variables and the outcomes assessed. Even after excluding subjects on medications that decrease serum UA, the possibility of a reverse causality must not be ruled out.

In conclusion, our findings suggest that dietary intake of some food groups (such as dairy, meats, beer and alcoholic beverages) seem to independently influence serum UA levels and hyperuricemia in different directions, which reinforces some recommendations, particularly on the potential benefit of a dairy-rich diet and low intake of beer and spirits. We believe that new studies on this subject should focus on dietary patterns instead of food groups or separate food items alone, and perform sex-stratified analyses, considering the differences that usually exist in food quantities, types and combinations consumed by men and women.

\section{Acknowledgements}

Acknowledgements: We would like to thank all ELSA-Brasil participants for their valuable contribution to this study. Financial support: The ELSA-Brasil baseline study is supported by the Brazilian Ministry of Health, Science and Technology Department (DECIT); and the Brazilian Ministry of Science and Technology and Innovation, Financiadora de Estudos e Projetos (FINEP) and Conselho Nacional de Desenvolvimento Científico e Tecnológico (CNPq) (grant numbers 0106 0010.00 RS, 0106 0212.00 BA, 0106 0300.00 ES, 0106 0278.00 MG, 0106 0115.00 SP and $01060071.00 \mathrm{RJ})$. SMB is a research fellow of the National Research Council - CNPq/Brazil (grant no. 300159/99-4) and the Fundação Estadual de Amparo da Pesquisa - FAPEMIG/Brazil (grant no: PPM-00274-17). MTS was supported by the Brazilian Coordination for the Improvement of Higher Education Personnel (CAPES) Finance Code 001. The funders had no role in study design, data collection and analysis, decision to publish, or preparation of the manuscript. Conflict of interest: None. Authorship: M.T.S., M.F.H.S.D., and S.M.B. designed the research project; M.T.S., M.F.H.S.D. and S.M.B. analysed the data; M.T.S., M.F.H.S.D., C.G.C., R.W.T. and S.M.B.wrote the paper; P.G.V. revised the manuscript. M.T.S., M.F.H.S.D., C.G.C., R.W.T. and S.M.B. had primary responsibility for final content. All authors read and approved the final manuscript. Ethics of buman subject participation: This study was conducted according to the guidelines laid down in the Declaration of Helsinki and approved by the Committee of Ethics in Research (approval number 189/2006). Written informed consent was obtained from all participants. 


\section{Supplementary material}

To view supplementary material for this article, please visit https://doi.org/10.1017/S1368980019002490

\section{References}

1. Perez-Ruiz F, Dalbeth N \& Bardin T (2015) A review of uric acid, crystal deposition disease, and gout. Adv Ther 32, 31-41.

2. Ben SC, Slim R, Fathallah N et al. (2017) Drug-induced hyperuricaemia and gout. Rheumatology 56, 679-688.

3. Bardin T \& Richette P (2014) Definition of hyperuricemia and gouty conditions. Curr Opin Rheumatol 26, 186-191.

4. Roddy E \& Choi HK (2014) Epidemiology of gout. Rheum Dis Clin North Am 40, 155-175.

5. Gropper SS, Smith JL \& Groff JL (2011) Nutrição avançadae metabolismo bumano. São Paulo: Cengage Learning.

6. Oliveira EP \& Burini RC (2012) High plasma uric acid concentration: causes and consequences. Diabetol Metab Syndr $\mathbf{4}$, $4-12$.

7. Gustafsson D \& Unwin R (2013) The pathophysiology of hyperuricaemia and its possible relationship to cardiovascular disease, morbidity and mortality. BMC Nephrol 14, 1-9.

8. Tsumuraya Y, Hirayama T, Tozuka E et al. (2015) Impact of hyperuricaemia on the chronic kidney disease-associated risk factors in a community-based population. Nephrol 20, 399-404.

9. Yan D, Tu Y, Jiang F et al. (2015) Uric acid is independently associated with diabetic kidney disease: a cross-sectional study in a Chinese population. Plos One 10, 1-9.

10. Reginato AM, Mount DB, Yang I et al. (2012) The genetics of hyperuricaemia and gout. Nat Rev Rheumatol 10, 610-621.

11. Babiker M (2015) Intake of purine-rich foods, total meat, seafood and dairy products and relationship to serum of uric acid. Am J Biomed Sci 14, 35-43.

12. Zykova SN, Storhaug HM, Toft I et al. (2015) Cross-sectional analysis of nutrition and serum uric acid in two Caucasian cohorts: The AusDiab Study and the Troms $\varnothing$ Study. Nutr J 14, 1-11.

13. Guasch-Ferré M, Bulló M, Babio N et al. (2013) Mediterranean diet and risk of hyperuricemia in elderly participants at high cardiovascular risk. J Gerontol A Biol Sci Med Sci 68, 1263-1270.

14. Choi HK \& Curhan G (2004) Beer, liquor, and wine consumption and serum uric acid level: the Third National Health and Nutrition Examination Survey. Arthritis Rheum 51, 1023-1029.

15. Kontogianni MD, Chrysohoou C, Panagiotakos DB et al. (2012) Adherence to the mediterranean diet and serum uric acid: the ATTICA study. Scand J Rheumatol 41, 442-449.

16. Schmidt JA, Crowe FL, Appleby PN et al. (2013) Serum uric acid concentrations in meat eaters, fish eaters, vegetarians and vegans: a cross-sectional analysis in the EPIC-Oxford cohort. Plos One 8, 1-8.

17. Zgaga L. Theodoratou E, Kyle J et al. (2012) The association of dietary intake of purine-rich vegetables, sugar-sweetened beverages and dairy with plasma urate, in a cross-sectional study. Plos One 7, 1-8.

18. Kaneko K, Aoyagi Y, Fukuuchi T et al. (2014) Total purine and purine base content of common foodstuffs for facilitating nutritional therapy for gout and hyperuricemia. BiolPharm Bull 37, 709-721.
19. Tsai Y, Liu JP, Tu YK et al. (2012) Relationship between dietary patterns and serum uric acid concentrations among ethnic Chinese adults in Taiwan. Asia Pac J Clin Nutr 21, 263-270.

20. Yu K-H, See LC, Huang YC et al. (2008) Dietary factors associated with hyperuricemia in adults. Semin Arthritis Rheum 37, 243-350.

21. Oliveira EP, Moreto F, Silveira LVA et al. (2013) Dietary, anthropometric, and biochemical determinants of uric acid in free-living adults. Nutr J 12, 1-10.

22. Aquino EML, Barreto SM, Bensenor IM et al. (2012) Brazilian longitudinal study of adult health (ELSA-Brasil): objectives and design. Am J Epidemiol 175, 315-324.

23. Schmidt MI, Duncan BB, Mill JG et al. (2015) Cohort profile: longitudinal study of adult health (ELSA-Brasil). Int $J$ Epidemiol 44, 68-75.

24. Fedeli LG, Vidigal PG, Leite CM et al. (2013) Logística de coleta e transporte de material biológico e organização do laboratório central no ELSA-Brasil. Rev Saúde Pública 47, 63-71.

25. Barreto SM, Ladeira RM, Duncan BB et al. (2016) Chronic kidney disease among adult participants of the ELSA-Brasil cohort: association with race and socioeconomic position. J Epidemiol Community Health 70, 380-389.

26. Molina Mdel CB, Faria CP, Cardoso LO et al. (2013) Diet assessment in the Brazilian Longitudinal Study of Adult Health (ELSA-Brasil): development of a food frequency questionnaire. Rev Nutr 26, 167-176.

27. Drehmer M. Pereira MA, Schmidt MI et al. (2016) Total and full-fat, but not low-fat, dairy product intakes are inversely associated with metabolic syndrome in adults. $J$ Nutr $\mathbf{1 4 6}$, 81-89.

28. Beydoun MA, Canas J-A, Fanelli-Kuczmarski MT et al. (2017) Genetic risk scores, sex and dietary factors interact to alter serum uric acid trajectory among African-American urban adults. Br J Nutr 117, 686-697.

29. Fardet A \& Rock E (2018) In vitro and in vivo antioxidant potential of milks, yoghurts, fermented milks and cheeses: a narrative review of evidence. Nutr Res Rev 31, 52-70.

30. Garrel DR, Verdy M, PetitClerc C et al. (1991) Milk - and soy - protein ingestion: acute effect on serum uric acid concentration. Am J Clin Nutr 53, 665-669.

31. Chang W-C (2011) Dietary intake and the risk of hyperuricemia, gout and chronic kidney disease in elderly Taiwanese men. Aging Male 14, 195-202.

32. Vilela NB \& Rocha R (2008). Manual básico para atendimento ambulatorial em nutrição, 2nd ed., Salvador: Editora da Universidade Federal da Bahia.

33. Choi HK, Mount DB \& Reginato AM (2005) Pathogenesis of gout. Ann Inter Med 143, 499-516.

34. Burns CM \& Wortmann RL (2012) Latest evidence on gout management: what the clinician needs to know. Ther Adv Chronic Dis 3, 271-286.

35. Choi HK, Atkinson K, Karlson EW et al. (2004) Purine-rich foods, dairy and protein intake, and the risk of gout in men. $N$ Engl J Med 350, 1093-1103.

36. Cardoso LO, Carvalho MS, Cruz OG et al. (2016) Eating patterns in the Brazilian Longitudinal Study of Adult Health (ELSA-Brasil): an exploratory analysis. Cad Saúde Pública 32. Published online: 13 May 2016. doi: 10.1590/0102311X00066215

37. Molina Mdel C, Benseñor IM, Cardoso LO et al. (2013) Reproducibility and relative validity of the Food Frequency Questionnaire used in the ELSA-Brasil. Cad Saúde Pública 29, 379-389. 